\title{
Is There Unification in the 21st Century?
}

\author{
Yuan K. Ha \\ Department of Physics, Temple University \\ Philadelphia, Pennsylvania 19122 U.S.A. \\ yuanha@temple.edu
}

July 1, 2010

\begin{abstract}
In the last 100 years, the most important equations in physics are Maxwell's equations for electrodynamics, Einstein's equation for gravity, Dirac's equation for the electron and Yang-Mills equation for elementary particles. Do these equations follow a common principle and come from a single theory? Despite intensive efforts to unify gravity and the particle interactions in the last 30 years, the goal is still to be achieved. Recent theories have not answered any question in physics. We examine the issues involved in this long quest to understand the ultimate nature of spacetime and matter.
\end{abstract}




\section{Introduction}

At the end of the 19th century, physicists were very confident that they had the laws of nature at hand. Classical mechanics had been firmly established for 200 years. Celestial mechanics was highly developed. Electrodynamics was discovered. Thermodynamics was understood. The euphoria was so evident that in a speech given by Albert Michelson in 1894 the following remarks were said [1]:

"The more important fundamental laws and facts of physical science have all been discovered, and these are so firmly established that the possibility of their ever being supplanted in consequence of new discoveries is exceedingly remote... Our future discoveries must be looked for in the sixth place of decimals."

Since then, physicists have identified four fundamental interactions together with their interaction strengths:

1. Electromagnetic interactions $-10^{-2}$.

2. Weak interactions $-10^{-5}$.

3. Strong interactions $-10^{0}$.

4. Gravitational interactions $-10^{-38}$.

They have also discovered a set of fundamental particles: the quarks $(u, d, s, c, b, t)$; the leptons $\left(e, \mu, \tau, \nu_{e}, \nu_{\mu}, \nu_{\tau}\right)$; the gauge bosons $\left(\gamma, W^{+}, W^{-}, Z^{0}, G^{\alpha \beta}\right)$, and the still anticipated Higgs boson $H^{0}$. These interactions and particles are governed by four fundamental equations:

1. Maxwell's equations (1864).

2. Einstein's equation (1915).

3. Dirac's equation (1928).

4. Yang-Mills equation (1954). 
The theories constructed for the fundamental interactions are gauge theories based on various Lie groups:

1. Quantum Electrodynamics - $U(1)$.

2. Quantum Electroweak Theory - $S U(2) \times U(1)$.

3. Quantum Chromodynamics - $S U(3)$.

4. Classical General Relativity - $S O(3,1)$.

The goal of unification is not simply to combine the various fundamental interactions in a consistent mathematical framework. It should entail a unifying principle and produce an interlocking structure. It should answer longstanding questions and make new predictions. The search for unification would force physicists to confront fundamental issues, to abandon old dogmas and to recognize new realities. The quantum theory of elementary particles has been quite successful in that it can explain accurately a number of phenomena in the strong, weak and electromagnetic interactions. On the other hand, it is gravity which is the most challenging and the least understood of the four interactions. We shall therefore focus our attention in this article to the problems in gravity. Without a deeper understanding of the nature of gravity and the theories which claim to explain it, unification is pointless as recent attempts to unify the interactions have not answered any question in physics.

\section{$2 \quad$ Supersymmetry}

The goal of supersymmetry is to unify spacetime and internal symmetries of elementary particles, thereby evading the Coleman-Mandula theorem which states that all possible symmetries of the S-matrix under general assumptions can only be a direct product of the Poincare algebra and an internal symmetry algebra. In supersymmetry, there exists a symmetry between fermions and bosons and the prediction is for every boson there exists a corresponding fermion of the same mass and quantum numbers. The role of supersymmetry is to cancel divergences in the perturbative calculations of quantum field the- 
ory since fermion and boson have opposite signs in loop corrections. In the standard model, quadratic corrections to the Higgs mass due to Yukawa interactions appear that cause its mass to diverge. Supersymmetry does away with the corrections by supplying terms with a minus sign. This scheme works through some physical cutoff mechanism, and there is a scale associated with it. Within the dimensional regularization approach, however, quadratic divergences do not exist and it is not clear what purpose would be served by a supersymmetric theory. From another point of view, the goal of supersymmetry is not to double the number of fundamental parti-

cles. The doubling of particles has already been achieved by the existence of antiparticles. Antiparticles are crucial in virtual particle pair creations and annihilations in quantum field theory. So far there is no irrefutable evidence that supersymmetry is a symmetry of nature after 40 years. According to Veltman [2]:

"The concept of naturalness is usually cited as the underlying motivation for supersymmetry. We will challenge that concept, and in any case need to point out that there is nothing natural about the development of the theory itself. Its main success is its agility in dodging the facts. The dubious explanation of the convergence of the three scale coupling constants into a single point can not be taken seriously. It is just another fit, using some of the many free parameters."

It should be pointed out that coupling constant unification does not prove unification of the strong, weak and electromagnetic interactions. There are other particles that can produce coupling constant unification.

\section{$3 \quad$ Higher Dimensions}

Many unification theories involve higher spacetime dimensions. There is nothing compelling about higher dimensions themselves. They may simply be a book-keeping device to account for the number of observed gauge fields. Gauge transformations are coordinate transformations in higher-dimensional 
space. In general, higher-dimensional theories suffer from instability and causality problems. There are negative energy solutions of the field equation. In Kaluza-Klein type theories of pure gravity in higher dimensions, the difficulty is noticeable at the classical level. Analysis of the perihelion shift of planets in the solar system shows that the shift depends on the total number of spatial dimensions in these theories [3]. The decomposition of metric assumes only a compact internal space with the geometry of tori. The result is independent of the size of the extra dimensions, even if it is of sub-millimeter scale. Starting from the multidimensional Einstein equation, a nonrelativistic limit of the metric in four dimensions can be obtained. The metric coefficients are found to depend explicitly on the total number of spatial dimensions $D$ and they affect the equation of motion in general relativity. In the perihelion shift calculation of the planet Mercury, the resulting formula is given by

$$
\frac{D}{D-2}\left(\frac{\pi m^{2} c^{2} R_{S}^{2}}{2 M^{2}}\right),
$$

where $m$ is the mass of the planet; $M$, the mass of the Sun; $R_{S}$, the Schwarzschild radius of the Sun, and $c$ is the speed of light. The observed discrepancy for Mercury is $43.11 \pm 0.21$ arcsec per century. Only the ordinary three-dimensional case $D=3$ gives a satisfactory result $42.94^{\prime \prime}$ which is within the measurement accuracy. For $D=4$, the result is $28.63^{\prime \prime}$ and for $D=9$ it is $18.40^{\prime \prime}$. Thus all multidimensional case $D>3$ contradict observations.

In the deflection of light by the Sun, a corresponding analysis provides the formula [4]

$$
\frac{D-1}{D-2}\left(\frac{R_{S}}{R}\right)
$$

where $R$ is the radius of the Sun. The observed deflection of a light ray that grazes the Sun surface has a historical value of 1.75 arcsec. For the threedimensional case $D=3$ the above formula reproduces this value accurately. For $D=4$, the result is $1.31^{\prime \prime}$ and for $D=9$ it is $1.00^{\prime \prime}$. Again, the multidimensional case shows a severe problem with the classical tests of general 
relativity. The implication of incorporating Kaluza-Klein type theories in unification is rather obvious.

\section{Higher Derivative Gravity Theories}

A number of theories known as higher derivative gravity theories have the goal of constructing a renormalizable theory of gravity explicitly in four dimensions. The Lagrangians contain higher order curvature invariants in Riemannian geometry such as those of scalar curvature $R^{2}$, Ricci curvature $R_{\alpha \beta} R^{\alpha \beta}$, Riemann curvature $R_{\alpha \beta \mu \nu} R^{\alpha \beta \mu \nu}$ and other combinations of these terms, including Weyl curvature invariant $C_{\alpha \beta \mu \nu} C^{\alpha \beta \mu \nu}$, in order that the equations be invariant under general coordinate transformations. These theories generally have problems with stability, unitarity, ghosts and nonlocality [5]. None of them is yet successful as a quantum theory of gravity. A further problem of higher derivative theories at the classical level is that none admits Birkhoff's theorem [6], which states that spherically symmetric solution is unique and time-independent. The failure of Birkhoff's theorem in higher derivative gravity theories means that spherically symmetric solution is time-dependent and dynamical. A similar failure of Birkhoff's theorem in a generalization of Einstein's gravity called $f(R)$ theory, in which the action is a nonlinear function of the scalar curvature $R$, also shows that spherically symmetric solutions are time-dependent [7]. As a result, black holes in these theories are dynamical. Their horizons disappear and a naked singularity will emerge [8]. In some $f(R)$ models, relativistic stars cannot exist due to the dynamics of the effective scalar degree of freedom and there are doubts about the viability of these models [9].

\section{$5 \quad$ Alternative Gravity Theories}

There are still other efforts to modify Einstein's gravity theory in order to achieve a finite and consistent theory of quantum gravity. These are generally known as modified gravity theories [10]. The modification can take place 
both at the microscopic scale and at the macroscopic scale [11]. At very small distances near the Planck length, modifications have included discrete spacetime, breaking of discrete symmetries, Lorentz symmetry violation, nonlocal interaction, extra dimensions, and non-commutative coordinates. However, these modifications are extremely tiny to be noticed at their current observational levels. Decoupling at the Planck scale prevents these effects from being observed at low energies. At large distances, modifications have included varying speed of light, varying gravitational constant, modifying Newton's Second Law of motion, non-symmetric metric and incorporating scalar, vector, and tensor particles into Einstein's gravity. The difficulty at this end is to obtain agreement with all astrophysical and solar system observations. So far none of these alternative theories of gravity has succeeded in replacing general relativity as the best theory of gravity.

A more fruitful approach to understand gravity is to develop quantum field theory of particles in curved spacetime [12]. This is done by treating spacetime classically and matter fields quantum mechanically. It is possible to study particle creation in strong gravitational fields. This has led to the prediction of Hawking radiation in which particles are emitted from a black hole with a thermal spectrum [13]; the Unruh effect in which an observer under acceleration in vacuum sees a thermal collection of particles [14]; and interpreting Einstein's equation as a thermodynamic equation of state of spacetime and matter [15], thereby realizing toward an emergent theory of gravity [16].

\section{$6 \quad$ Is Spacetime Quantum?}

In special relativity, the Lorentz transformation is a pseudo-rotation in fourdimensional Minkowski spacetime. It is not possible to include the Planck constant or any other parameter into the transformation. It is a purely mathematical transformation. Therefore there is no such theory to be called quantum theory of special relativity. This term has a completely differ-

ent meaning from relativistic quantum mechanics which is a description of 
matter. Similarly, it is not possible to include Planck's constant in general coordinate transformations, or to have a quantum theory of Riemannian spacetime. Since geometry is gravity in general relativity, this calls into the question whether gravity really needs to be quantized [17][18]. Spacetime originally is a macroscopic concept. Is it possible that Einstein's equation is similar in nature to Navier-Stokes equation in fluid mechanics as a macroscopic theory $[19]$ ?

The investigation of quantum black holes [20] shows that they are extremely microscopic objects with a macroscopic mass. Their Schwarzschild radius is equal to their Compton wavelength. They exist at the boundary between classical and quantum regions. They obey the Laws of Thermodynamics and they decay into elementary particles. A quantum black hole of the size of the Planck length $1.6 \times 10^{-33} \mathrm{~cm}$ has a mass of $2.2 \times 10^{-5}$ gm. Like the nucleus of a heavy atom, quantum black holes may require the use of quantum mechanics but not necessarily quantum field theory for their description. The difference between quantum mechanics and quantum field theory is tremendous - it is the creation and annihilation of particles. There are no anti-black holes in general relativity. Therefore there are no virtual pair creations and annihilations of black holes as in ordinary particles. Two black holes combine to form another black hole according to the area non-decrease theorem. The resulting black hole evaporates according to Hawking's description with a temperature. Quantum black holes are intrinsically semi-classical objects.

\section{Quantum Gravity In Crisis}

An important result in cosmology was obtained recently which can elucidate the nature of spacetime down to the smallest scale. This is the observation of the highest energy gamma rays from a gamma ray burst GRB 090510 by the Fermi Gamma-Ray Space Telescope [21]. A single 31-GeV photon was detected from a source at a redshift of $z=0.903$ which corresponds to a distance of 7.3 billion light years from Earth. It was the last of the seven 
pulses in a short burst that lasted for $0.829 \mathrm{~s}$. One of the two postulates of Einstein's special relativity is Lorentz invariance in that all observers measure exactly the same speed of light in vacuum, independent of the motion of the source and of the photon energy. In certain quantum theories of gravity, there is great interest in the possibility that Lorentz invariance might be broken near the Planck scale due to quantum fluctuation of spacetime and the notion of spacetime foam. A variation of photon speed is an indication that Lorentz invariance is violated. This may be revealed by observing the sharp features in the gamma ray burst light-curves. If the spread in travel time of less than $0.9 \mathrm{~s}$ between the highest and lowest-energy gamma rays in the burst GRB 090510 is all attributed to quantum effects, then a thorough analysis shows that any quantum effects in which the speed is linearly proportional to energy do not show up until the distance is down to about $0.8 L_{P l}$, which is below the Planck length. This result therefore rules out a number of quantum gravity models that predict such linear variation with energy.

The gamma ray burst reported above is significant in that it allows for the exploration of spacetime near Planck length by using effects accumulated over cosmological distances since direct access to Planck energy in experiments is not possible. The result indicates that there is no evidence so far of any quantum nature of spacetime above the Planck length. Spacetime there is smooth and continuous. The speed of light is constant and special relativity is right. At the Planck length, quantum black holes would appear in observation and they effectively provide a natural cutoff to spacetime. For observable purpose, it is not necessary to consider theories below the Planck length. Further detections using gamma ray bursts with even higher energy photons will settle the question of quantum spacetime definitively. It would be amazing that in effect spacetime is classical and there is no need for a quantum theory of gravity. There would be an underlying theory for gravity which is not gravity, just as statistical mechanics is the underlying theory of thermodynamics. Unification would have a very different meaning from the current understanding involving quantum gravity as a fundamental premise. 


\section{References}

[1] A. Michelson - Opening of Ryerson Laboratory at University of Chicago.

[2] M. Veltman, Acta Phys. Pol. B 25, 1399 (1994).

[3] M. Eingorn and A. Zhuk, arXiv: 0912.2698.

[4] M. Eingorn and A. Zhuk, arXiv: 1003.5690.

[5] T. Chiba, JCAP 03, 008 (2005).

[6] P. Havas, Gen. Rel. Grav. 8, 631 (1977).

[7] V. Faraoni, Phys. Rev. D 81, 044002 (2010).

[8] V. Faraoni, arXiv: 1005.5398.

[9] T. Kobayashi and K. Maeda, Phys. Rev. D 78, 064019 (2008).

[10] J.W. Moffat, Reinventing Gravity (HarperCollins, New York, 2008).

[11] C.P. Burgess, arXiv: 0912.4295.

[12] R.M. Wald, Quantum Field Theory in Curved Spacetime and Black Hole Thermodynamics (The University of Chicago Press, Chicago, 1994).

[13] S.W. Hawking, Commun. Math. Phys. 43, 199 (1975).

[14] W.H. Unruh, Phys. Rev. D 14, 870 (1976).

[15] T. Jacobson, Phys. Rev. Lett. 75, 1260 (1995).

[16] T. Padmanabhan, AIP Conf. Proc. 939, 114 (2007).

[17] S. Carlip, Class. Quant. Grav. 25, 154010 (2008).

[18] S. Boughn, Found. Phys. 39, 331 (2009).

[19] Y.K. Ha, Int. J. Mod. Phys. A 24, 3577 (2009).

[20] Y.K. Ha, arXiv: 0812.5012.

[21] A.A. Abdo et al., Nature 462, 331 (2009). 